\title{
Beam diagnostics for low energy beams
}

\author{
J. Harasimowicz* and C. P. Welsch \\ Cockcroft Institute, Warrington WA4 4AD, United Kingdom, \\ and Department of Physics, University of Liverpool, Liverpool L69 7ZE, United Kingdom
}

L. Cosentino, A. Pappalardo, and P. Finocchiaro

National Institute of Nuclear Physics INFN-LNS, Catania 95125, Italy

(Received 23 July 2012; published 11 December 2012)

\begin{abstract}
Low-energetic ion and antimatter beams are very attractive for a number of fundamental studies. The diagnostics of such beams, however, is a challenge due to low currents down to only a few thousands of particles per second and significant fraction of energy loss in matter at keV beam energies. A modular set of particle detectors has been developed to suit the particular beam diagnostic needs of the ultralowenergy storage ring (USR) at the future facility for low-energy antiproton and ion research, accommodating very low beam intensities at energies down to $20 \mathrm{keV}$. The detectors include beam-profile monitors based on scintillating screens and secondary electron emission, sensitive Faraday cups for absolute intensity measurements, and capacitive pickups for beam position monitoring. In this paper, the design of all detectors is presented in detail and results from beam measurements are shown. The resolution limits of all detectors are described and options for further improvement summarized. Whilst initially developed for the USR, the instrumentation described in this paper is also well suited for use in other low-intensity, low-energy accelerators, storage rings, and beam lines.
\end{abstract}

DOI: 10.1103/PhysRevSTAB.15.122801

\section{INTRODUCTION}

A next-generation facility for low-energy antiproton and ion research (FLAIR) will provide world-wide unique conditions for experiments with cooled low-energy antiprotons [1]. Its central machine, the ultralow-energy storage ring (USR) [2], will be able to accept $300 \mathrm{keV}$ particles and decelerate them to $20 \mathrm{keV}$ or possibly to even lower energies. The ring was designed to offer a wide range of beam configurations, ranging from very short pulses in the nanosecond regime to a quasi-DC beam. Additionally, it features a combined fast and slow extraction scheme that can provide external experiments with cooled beams of various time structures. Because of the space charge limitation, approximately $2 \times 10^{7}$ single-charged $20 \mathrm{keV}$ particles can be stored [3], but the effective antiproton rates for in-ring experiments can reach even $10^{12}$ particles per second (pps). With a slow extraction scheme, as few as $5 \times 10^{5}-10^{6} \mathrm{pps}$, corresponding to beam currents of approximately $100 \mathrm{fA}$, are expected. With fast extraction, all antiprotons will be ejected into traps within a time shorter than $200 \mathrm{~ns}$. Table I summarizes the most important USR beam parameters. Although antiprotons are a focus at FLAIR, other particles, such as protons or $\mathrm{H}^{-}$ions, will be used for the initial commissioning of the machine.

\footnotetext{
*Janusz.Harasimowicz@liverpool.ac.uk
}

Published by the American Physical Society under the terms of the Creative Commons Attribution 3.0 License. Further distribution of this work must maintain attribution to the author(s) and the published article's title, journal citation, and DOI.
PACS numbers: 07.77.Ka, 29.40.Gx, 29.27.-a, 13.75.-n

The boundary conditions of the USR put challenging demands on its beam instrumentation. Most of the standard diagnostic solutions for high-energy conditions no longer work (e.g. [4-6]), necessitating significant new development efforts. First, low-energy particles can be easily disturbed and any intercepting solution will result in the emittance blowup and, eventually, beam loss. For instance, $300 \mathrm{keV}$ protons traversing an aluminum layer as thin as $500 \mathrm{~nm}$ undergo multiple scattering and lose more than $40 \mathrm{keV}$ of their kinetic energy, whereas $20 \mathrm{keV}$ protons are already fully stopped in the same layer [7]. Second, the limited number of particles in the USR results in ultralow intensities to be measured. Approximately $10^{7}$ protons or antiprotons circulating in the ring correspond to a few hundred nanoamperes, well below the detection limits of standard beam-current transformers used in highenergy accelerators [8]. The same number of particles passing only once through a transfer line generates average currents in the femtoampere range. These are difficult to measure even outside an accelerator environment [9]. Third, although antiprotons are of the main interest at the USR, also protons or $\mathrm{H}^{-}$ions will be used for the initial commissioning of the machine. However, stopped antiparticles generate completely different signals than ions. The energy released in annihilation of $\mathrm{keV}$ antiprotons is a few orders of magnitude higher than the energy deposited by $\mathrm{keV}$ protons [10]. Among various types of secondary particles, high-energy pions and strongly ionizing ions are created and constitute an important contribution to the response of a detector. 
TABLE I. General parameters of antiproton beams at the USR.

\begin{tabular}{lc}
\hline \hline Beam energy & $20 \mathrm{keV}-300 \mathrm{keV}$ \\
Relativistic $\beta=v / c$ & $0.006-0.025$ \\
Revolution frequency & $46 \mathrm{kHz}-178 \mathrm{kHz}$ \\
Revolution time & $5.6 \mu \mathrm{s}-21.8 \mu \mathrm{s}$ \\
Number of particles & $\leq 2 \times 10^{7} @ 20 \mathrm{keV}$ \\
(space charge limit) & \\
Bunch length & $1 \mathrm{~ns}-$ quasi-DC beam \\
Beam diameter & few mm-several cm \\
Effective in-ring antiproton rates & $10^{10} \mathrm{pps}-10^{12} \mathrm{pps}$ \\
Average rates of extracted & $5 \times 10^{5} \mathrm{pps}-10^{6} \mathrm{pps}$ \\
antiprotons & \\
\hline \hline
\end{tabular}

A modular set of high performance diagnostic tools was developed and optimized for the USR conditions. The devices comprise scintillating screens, secondary emission monitors, Faraday cups, and capacitive pickups. A flexible ionization monitor for minimally interceptive, transverse beam-profile measurements is also part of this instrumentation but will not be discussed in this paper; details are given in [11]. Although all monitors were optimized for the USR, their use stretches well beyond this particular machine and they are suited for other low-energy storage rings and beam lines as well.

\section{SCINTILLATING SCREENS}

In terms of simplicity, cheapness, and effectiveness, scintillators are among the best suited instruments for beamprofile monitoring. While in general they are not as sensitive as detectors equipped with amplification devices, see Sec. III, their ultimate detection limits at low energies have not been investigated in great detail [12]. A further challenge comes from the fact that the USR requires beam diagnostics for both, antiprotons and protons, and the choice of a suitable scintillating material becomes even more complicated.

Detection limits of various scintillating materials were investigated with low-energy, low-intensity proton beams and results were reported in detail [13], hence only a brief summary will be given. The measurements

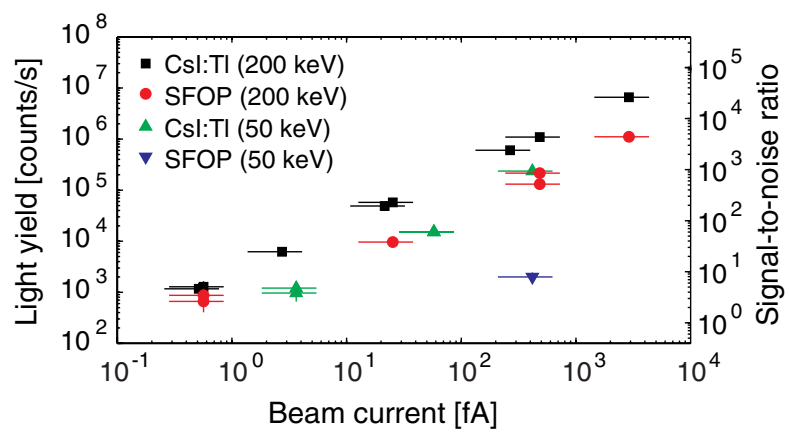

FIG. 1. Calibrated light output and signal-to-noise ratio as a function of beam current for CsI:Tl and SFOP, irradiated with 200 and $50 \mathrm{keV}$ proton beams [13]. were performed with the use of the Tandem accelerator at the National Institute of Nuclear Physics INFN-LNS in Catania [14]. Because the proton beams were delivered at 200 and $50 \mathrm{keV}$ with picoampere intensities, additional attenuating grids were introduced. It was demonstrated that cesium iodide doped with thallium (CsI:Tl) and a terbiumdoped glass scintillating fiber optic plate (SFOP) are sensitive enough to be applied to proton beam diagnostics at the USR. Their response to various beam energies and intensities is shown in Fig. 1. With $200 \mathrm{keV}$ beams, it was possible to measure currents even in the sub-fA range. At $50 \mathrm{keV}$, the sensitivity of both screens drops down and is about 4 times lower for CsI:Tl and approximately 2 orders of magnitude lower for the SFOP, respectively. It was shown that resolution of at least $0.3 \mathrm{~mm}$ can be achieved. Additionally, an absolute light yield calibration technique was successfully applied to facilitate beam-current estimates by future users.

Antiprotons impose additional challenges to the application of scintillators to beam-profile monitoring $[15,16]$. Stopped in a detector material, antiprotons annihilate and produce a variety of secondary particles. The annihilation products, mainly weakly ionizing, high-energy pions and recoiling, strongly ionizing $\mathrm{MeV}$-scale nuclear fragments, transfer enormous energy to the detector. As opposed to protons, the light yield due to the kinetic energy of antiprotons is only a small fraction of the light output due to the energy released after annihilation. As a result, two effects can be observed: saturation, caused by the amount of energy deposited locally, and ghost structures in the image due to secondary particles traveling through the scintillator and exciting electrons further away from the primary interaction point.

In spite of these shortcomings, a sensitive scintillating screen is still a detector of choice for the initial commissioning of the USR with protons or $\mathrm{H}^{-}$ions. A bulkscintillator based monitor was designed for this purpose and is shown in Fig. 2. It consists of a screen holder with an adjustable aperture attached to an actuator. Based on the sensitivity tests, CsI:Tl will be used as scintillating material. It is installed in a vacuum vessel at $45^{\circ}$ to the beam axis which enables observation of the light output by
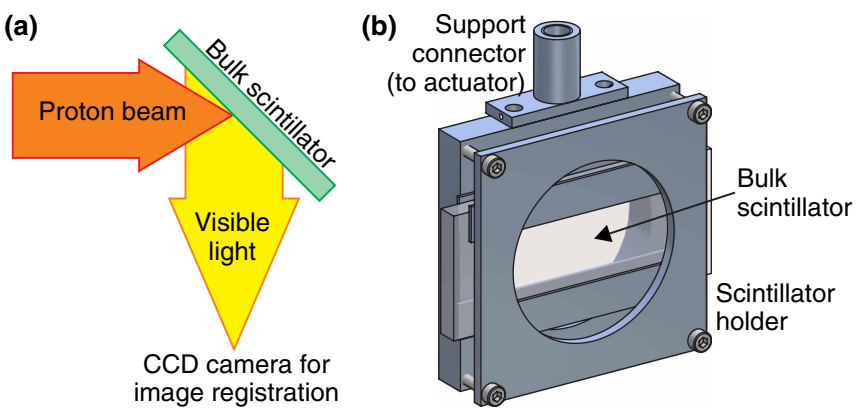

FIG. 2. Bulk-scintillator based profile monitor: (a) placement schematic, and (b) mechanical design for the USR. 
means of a charged coupled device (CCD) camera placed outside a vacuum window.

A profile monitor for antiproton beams should be optimized with the annihilation-related processes carefully taken into account. A scintillator suitable for ultralow-intensity proton beams, such as CsI:Tl, is expected to be too sensitive to highly ionizing annihilation products. Nevertheless, observation of antiproton beams is still possible using, for example, plastic or quartz glass as materials [16].

\section{FOIL-BASED SECONDARY EMISSION MONITOR}

\section{A. Motivation}

Electron multiplier microchannel plates (MCPs) offer a variable amplification and their applicability to antiproton monitoring has already been demonstrated [17]. Approximately $10^{5}$ antiprotons delivered in about $10 \mathrm{~ms}$ can be observed by an MCP placed directly in the beam path. However, higher amounts saturate the detector and can damage it in the process. Furthermore, an MCP placed directly in the beam is fully destructive, and for both reasons other configurations have been proposed (e.g. $[18,19])$. Their spatial resolution is limited to $1-2 \mathrm{~mm}$, but they offer sensitivity of tens or hundreds pps, variable gain, and are potentially less interceptive than bulk scintillators. Such solutions, however, have been used for measurements with either low-energy heavy ions or $\mathrm{MeV}$-scale protons which are not strongly disturbed by few $\mathrm{keV} / \mathrm{mm}$ accelerating gradients of the detectors.

One of the techniques to measure the beam profile with microchannel plates is based on the detection of secondary electrons emitted from a foil intercepting the primary beam. In such a system, the MCP is placed at some distance from the beam and only secondaries can reach its surface. They need to be guided by means of a high voltage applied to the foil, thus also the primary beam traverses a region of strong electric field. This is not a problem in high-energy or heavy-ion applications, but becomes crucial for $\mathrm{keV}$ proton or antiproton beams. Again, the annihilation of antiprotons results in emission of other high-energy particles which may affect the response of the monitor. Limitations of a foil-based secondary electron emission monitor (SEM) were investigated and its usability for $20-300 \mathrm{keV}$ beam imaging was tested.

\section{B. Principle of operation}

A foil-based SEM consists of a foil on negative potential and a grounded mesh, an MCP with a phosphor screen, and a CCD camera registering the image. Its schematic diagram is shown in Fig. 3.

The primary beam passes through the mesh at $45^{\circ}$ and ejects $\mathrm{eV}$-range secondary electrons from the foil surface. These secondaries are accelerated by the negative foil potential and fly through the mesh towards the detection system. There, they are multiplied by the MCP and

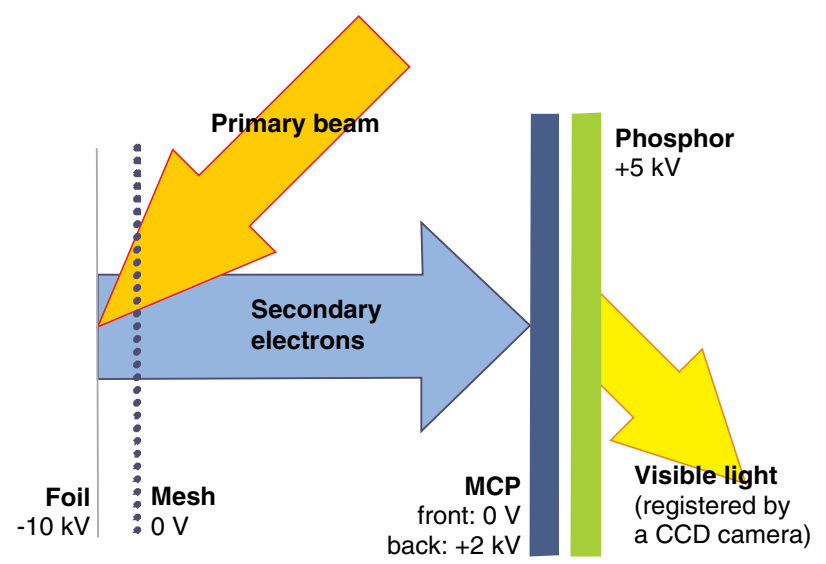

FIG. 3. Operation principle of a foil-based secondary emission monitor.

converted to visible light by a phosphor screen producing a beam image which can then be registered by a CCD camera.

\section{Design considerations}

A low-energy primary beam can be affected by the monitor in various ways: particles can lose their energy and eventually be stopped in the mesh and foil assembly, be scattered, or change their trajectories in the presence of electric field. These effects can cause beam loss, but also introduce strong distortions of the beam-profile image.

The mean path length for protons in aluminum is $3 \mu \mathrm{m}$ at $300 \mathrm{keV}$ and $0.3 \mu \mathrm{m}$ at $20 \mathrm{keV}$ [7], whereas for antiprotons it is about $4.5 \mu \mathrm{m}$ at $300 \mathrm{keV}$ and approximately $0.8 \mu \mathrm{m}$ at $20 \mathrm{keV}$ [20]. Because of multiple scattering effects, however, the penetration depth of particles is lower and for protons drops to $2.8 \mu \mathrm{m}$ at $300 \mathrm{keV}$ and $0.2 \mu \mathrm{m}$ at $20 \mathrm{keV}$ [7]. Consequently, a foil as thin as $200 \mathrm{~nm}$ makes the SEM a destructive beam monitor. The detector can be used in beam transfer lines and for first turn diagnostics, yet should be optimized for minimal image distortion due to electric fields and annihilation-related noise.

A model of the SEM was studied with SIMION [21]. The foil and mesh were approximated by two parallel circular disks of $50 \mathrm{~mm}$ diameter, separated by $5 \mathrm{~mm}$. A voltage of up to $-10 \mathrm{kV}$ was applied to the foil. The grounded mesh surface was made transparent to the beam. $50 \mathrm{~mm}$ away from the foil, a simplified MCP and phosphor assembly was located with 0 Volts and $2 \mathrm{kV}$ applied to the front and back of the microchannel plate, respectively, and $5 \mathrm{kV}$ applied to the scintillating screen. In order to minimize the influence of the electric fields of the SEM on the primary beams, additional shielding was included. Both the foil and the MCP/phosphor assembly were placed in grounded enclosures which also protected the back of the monitor from a direct beam hit. At $-10 \mathrm{kV}$ foil potential, a $20 \mathrm{keV}$ proton beam was deflected by $0.8 \mathrm{~mm}$ from its initial trajectory. Under the same conditions, almost the 
entire $20 \mathrm{keV}$ antiproton beam was bent away from the monitor before reaching the foil. With the potential reduced to $-5 \mathrm{kV}$, both beams hit the aluminum layer and their displacement from the center was 0.5 and $0.9 \mathrm{~mm}$ for protons and antiprotons, respectively. Although the shielding did not fully diminish the influence of the monitor on the primary beams, it made observations of negatively charged particles possible at reduced foil voltages.

In order to verify the capability of the monitor to image the primary beam profiles, the transport of secondary electrons from the foil to the detection system was studied as well. A Monte Carlo routine was written for SIMION to generate electrons at the foil surface and thus emulate secondary emission. A point source of electrons was defined and the particles were tracked through the electric field until stopped in the MCP. Their angular distribution was defined as a cosine distribution with the peak emission direction normal to the foil surface [22]. The energy distribution followed the spectrum for clean aluminum presented in [23]. Both distributions are shown in Fig. 4.

The theoretical resolution of the monitor was investigated by applying various voltages to the foil and recording the position of electrons hitting the MCP. Figure 5 illustrates the dependence of the image full width at half maximum (FWHM) as a function of the foil potential. The model predicts that a resolution no better than $2 \mathrm{~mm}$ at $-10 \mathrm{kV}$ can be obtained. Although not ideal, this is still
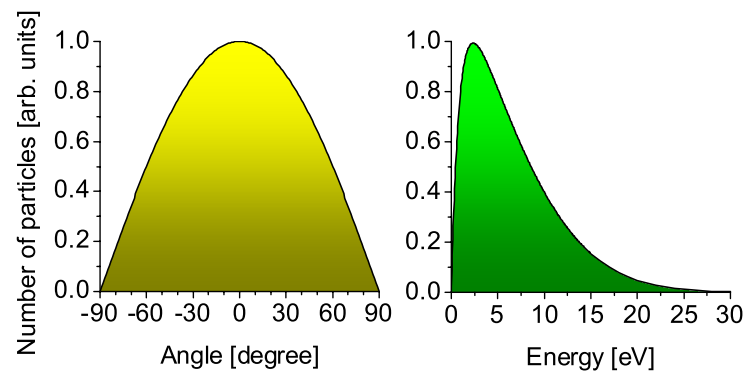

FIG. 4. Angular (left) and energy distribution (right) of simulated secondary electrons.

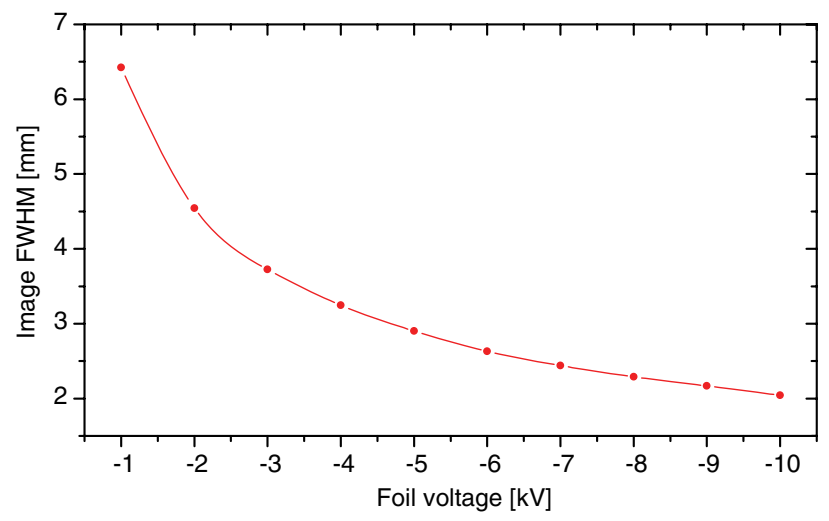

FIG. 5. Simulated FWHM of the images recorded at the MCP surface as a function of voltage applied to the foil. acceptable for beams of diameters up to a few centimeters provided by the USR.

In order to estimate the average number of electrons reaching the MCP surface, an assumption of the electron yield of roughly 1 particle per proton is made [22]. For a circular area of $20 \mathrm{~mm}$ diameter, this corresponds to approximately $3 \times 10^{-3}$ charges $/\left(\mathrm{mm}^{2} \cdot\right.$ proton $)$. However, secondary emission depends on a number of factors, such as material oxidation, purity, or surface roughness, which cannot be simulated with a high degree of accuracy.

It would be quite difficult to precisely model the behavior of the SEM bombarded with low-energy antiprotons. A combined charge signal from all of the effects due to annihilation was established $[10,24]$ but it would be rather impossible to implement in any precise way. Existing Monte Carlo codes can provide only a simplified picture of the processes inside the SEM without taking into account many substantial aspects, such as the creation of eV-scale secondaries. Most importantly, computer codes are limited by the experimental data for low-energy antiprotons. However, it is clear that the annihilation of antiprotons will increase the number of particles reaching the MCP and therefore will affect the observed image.

The FLUKA code $[25,26]$ was used to estimate the number of particles generated in a collision of $300 \mathrm{keV}$ antiprotons with a $100 \mu \mathrm{m}$ aluminum foil. A $20 \mathrm{~mm}$ diameter beam with a uniform distribution contained $2 \times 10^{7}$ antiprotons and was fully stopped in the foil placed at $45^{\circ}$. No additional materials or electric fields were introduced. According to the simulations, approximately $6 \times 10^{-5}$ charges $/\left(\mathrm{mm}^{2} \cdot\right.$ antiproton $)$ will reach the MCP surface. However, eV-scale particles were not considered.

\section{Prototype design}

The design of the USR beam-profile monitor was made flexible to enable the use of two configurations, the foilbased SEM and an MCP placed directly in the beam path, as shown in Fig. 6.

The MCP and phosphor are shielded to minimize the fringe field which could affect the low-energy beam, and to protect the back of the phosphor from scattered and halo particles. The foil and mesh are installed inside a grounded enclosure to minimize the disturbance of the beam as well. All the components are mounted on a pneumatic actuator.

The foil and mesh assembly consists of a $25 \mu \mathrm{m}$ thick aluminum foil which is separated from a nickel mesh by $5 \mathrm{~mm}$. The mesh is made of 80 wires per inch (about 3 wires per $\mathrm{mm}$ ) with each wire about $25 \mu \mathrm{m}$ thick and a pitch of about $293 \mu \mathrm{m}$.

The chevron-type MCP and the phosphor were procured as a single assembly from Beam Imaging Solutions [27] and are installed in a custom-made shield. The MCP channel diameter is $10 \mu \mathrm{m}$ with a pitch of $12 \mu \mathrm{m}$ and $8^{\circ}$ bias angle. The outer diameter is $50 \mathrm{~mm}$ with an active area diameter $>40 \mathrm{~mm}$. The thickness of each of the 

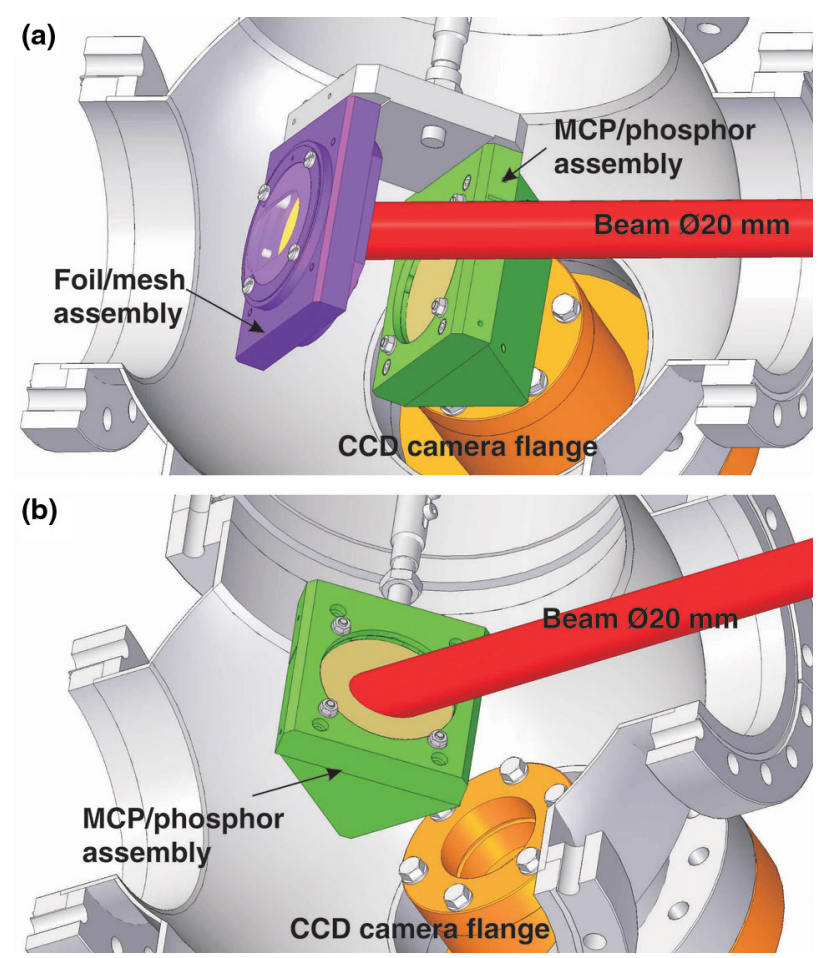

FIG. 6. Two configurations of the secondary emission monitor: (a) foil-based SEM, (b) MCP directly in the beam.

MCPs in the chevron stack is $0.46 \mathrm{~mm}$. The screen material used is aluminized P43 on glass. Its peak emitted wavelength is $545 \mathrm{~nm}$ which corresponds to green light.

\section{Experimental results}

The performance of the secondary emission monitor was investigated with $\mathrm{keV}$ protons available at INFN-LNS. The experimental setup is shown in Fig. 7. The $450 \mathrm{kV}$ injector of the Tandem accelerator was employed as a particle source. Downstream of the ion source and $90^{\circ}$ analyzing magnet, two pairs of $X$ and $Y$ variable slits were used as collimators. They were followed by an electrostatic Faraday cup, normally used for ion source mass analysis, and two removable pepper-pot grids for intensity reduction. Finally, two prototype monitors for the USR, a sensitive Faraday cup described in detail in Sec. IV of this paper

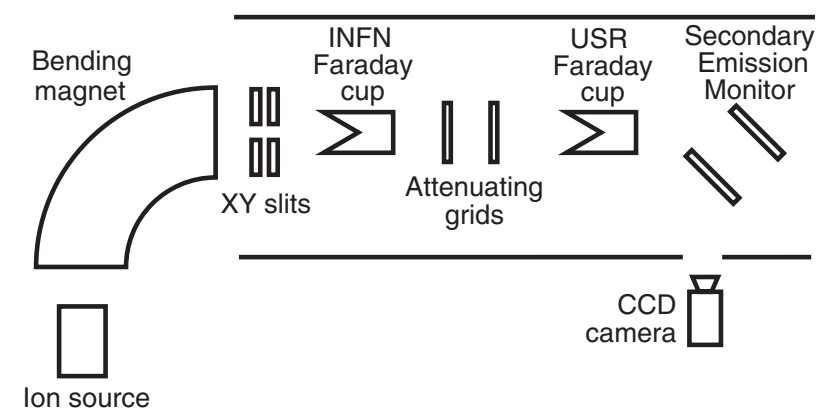

FIG. 7. Experimental setup for the secondary emission monitor tests at INFN-LNS. and the secondary emission monitor, were installed downstream of the attenuating grids. The vacuum level reached in the beam line was $10^{-7}$ mbar. Because of time constrains, only the foil-based configuration was tested and no images for the MCP placed directly in the beam path were obtained.

The beam energy was set to $200 \mathrm{keV}$ and its intensity was lowered to pA level. Femtoampere currents were obtained by defocusing the beam and employing both collimators and pepper-pot attenuators. Consequently, different intensities were observed at the beginning of the beam line and at the location of the USR prototypes a few meters away. It limited the application of the INFN-LNS Faraday cup as a reference monitor which was used for the initial beam setting, but not for absolute calibration.

Beam images were recorded with a high performance 14-bit CHROMA CX3 still camera produced by DTA, featuring a $1534 \times 1024$ pixel KAF1600 CCD, manufactured by Kodak. The device was installed in the in-vacuum viewport at $90^{\circ}$ to the beam axis, thus the back of the phosphor screen was observed at $45^{\circ}$. The camera was connected to a frame grabber computer board which enabled live display capture. The images were binned at $3 \times 3$ with an effective number of $512 \times 341$ pixels. Systematic noise, due to intrinsic nonuniformity of the CCD response and its readout, was reduced by taking an image under the same conditions, but with the shutter closed and subtracting it from a real image. This dark image subtraction was done automatically by the camera software.

Each beam image was analyzed according to the same procedure. A dark image had been automatically subtracted before any off-line analysis was started and IMAGEJ [28] was used for further processing. An IMAGEJ built-in filter was applied to the image to remove bad pixels visible even without the beam, i.e., intense pixels were replaced by an average of their neighbors. Afterwards, the intensity values of the two-dimensional (2D) picture were projected onto the $x$ axis and a Gaussian distribution was fitted. The result was used for beam width and center position determination. An image of the MCP and phosphor assembly was taken with reference markers at the back of the screen, which allowed translating the number of pixels to millimeters. This provided a calibration factor of $27.0 \pm 0.3$ pixels $/ \mathrm{mm}$. In addition, black-and-white images were color coded to present the $2 \mathrm{D}$ data in a clearer way.

The influence of the MCP gain on the image was investigated at fixed voltages applied to the foil and the phosphor screen. The foil was at $-10 \mathrm{kV}$, the phosphor at $5 \mathrm{kV}$, and the chevron MCP voltage varied between 1.6 and $2 \mathrm{kV}$. The result is shown in Fig. 8. The beam current measured by the INFN Faraday cup was about 50 pA, but only a fraction of the particles reached the SEM due to the use of two pepper-pot grids upstream the profile monitor. The beam current at the detector location was less than $20 \mathrm{fA}$ 


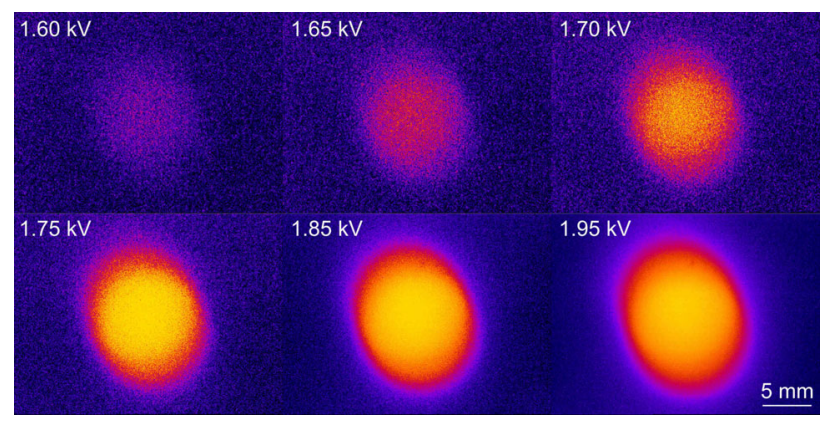

FIG. 8. Influence of the MCP gain on the image quality. See text for details.

as measured with the USR Faraday cup placed closer to the SEM. Because of a low number of particles, no images were observed below 1.6 kV MCP voltage. However, a clear, sharp image is seen at $1.85 \mathrm{kV}$, which means that at the highest voltage of $2 \mathrm{kV}$ even lower beam intensities can be monitored.

The transport of secondary electrons and its influence on the beam image were studied as well. In this case, the MCP voltage was set to $2 \mathrm{kV}$, the phosphor to $5 \mathrm{kV}$, and the voltage supplied to the foil varied from 0 to $-10 \mathrm{kV}$. Figure 9 shows a selection of color-coded 2D images.

The acquired 2D images were projected onto one axis and cumulative intensity distributions as a function of position were obtained and Gaussian profiles were fitted to them. The latter were used to calculate the peak center position and FWHM value at various foil voltages. The results are shown in Fig. 10.

The observed beam size, shown in Fig. 10(a), decreases with increasing voltage applied to the foil. The FWHM value reaches about $10 \mathrm{~mm}$ at the maximum voltage, but the width differs only slightly for lower voltages, which is in agreement with simulations. In addition, a variation of the center position can be noticed in Fig. 10(b). A large uncertainty of the center position determination for a weakly focused beam was assumed, thus its displacement was calculated relative to the position at $-1 \mathrm{kV}$. A significant variation in the projected beam image center for low voltages can be explained by a large size of the beam image

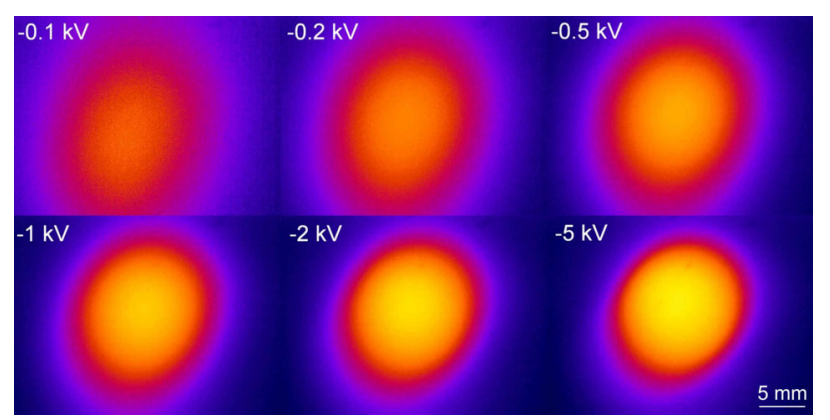

FIG. 9. Influence of the foil voltage on the image quality. See text for details.
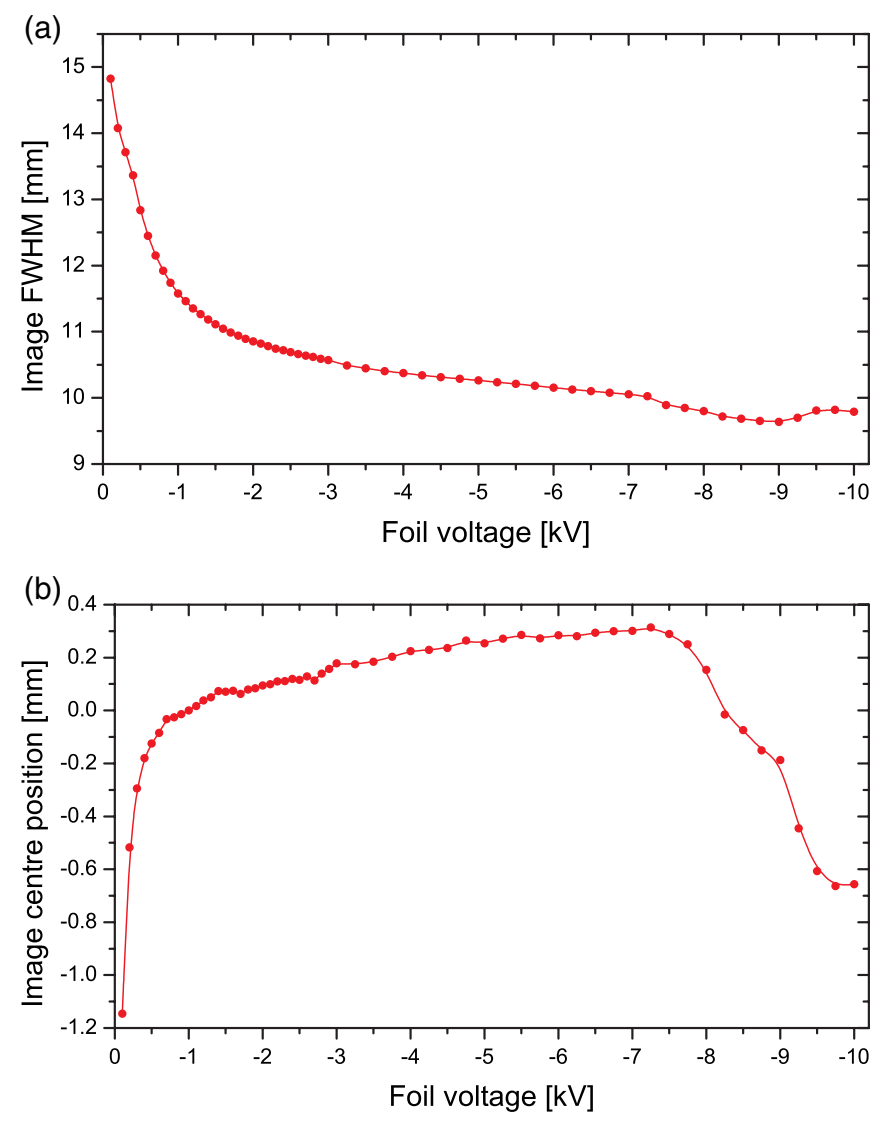

FIG. 10. Beam image FWHM (a) and center displacement relative to the beam position at $-1 \mathrm{kV}$ (b) as a function of the voltage applied to the foil.

and a blurred peak which becomes clearer only above a few hundred volts. Above $-500 \mathrm{~V}$ the discrepancy is smaller than $0.1 \mathrm{~mm}$. In the range between -1 and about $-7.5 \mathrm{kV}$, a steady yet small shift of the beam image center is observed reaching about $0.3 \mathrm{~mm}$ due to influence of the electric field on the primary beam. A different behavior was registered above $-7.5 \mathrm{kV}$ where both the image width and center position became disturbed with the beam shift reaching almost $0.7 \mathrm{~mm}$. This can be explained by a small deformation of the thin aluminum foil due to an electrostatic pressure caused by a high voltage applied to the system.

It is possible that the high potential difference between the foil and the mesh caused a noticeable deformation of the aluminum, leading to a change in the primary beam hit position. This is illustrated in Fig. 11. A simple assumption of a symmetric deflection of the foil clamped at the edges shows that the beam would hit a different point of the detector. For a $1 \mathrm{~mm}$ deformation, the shift of the beam image is approximately $1 \mathrm{~mm}$. However, nonlinear effects are expected for deflections larger than one-half the foil thickness [29]. Additionally, the foil was poorly stretched and was not ideally flat. The issue is subject to further analysis and measurements. 


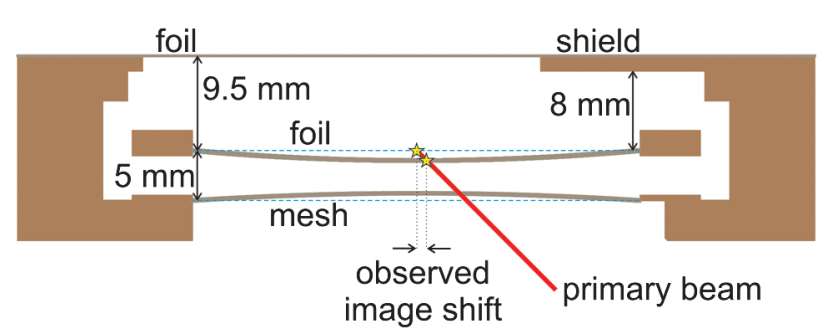

FIG. 11. Simplified deflection of the foil clamped at the edges and subject to a uniform electrostatic pressure. See text for details.

Finally, a collimator was prepared to investigate into the resolution of the monitor. A set of holes, 2 and $3 \mathrm{~mm}$ in diameter, were drilled in a $5 \mathrm{~mm}$ thick aluminum plate as shown in Fig. 12. The collimator was attached to the back of the MCP and phosphor assembly in order not to block the path of secondary electrons. As a consequence, the distance between the exit of the collimator and the center of the foil reached $76 \mathrm{~mm}$.

Beam images obtained with the collimator in place for various beam steering settings are shown in Fig. 13. The beam size upstream the collimator was not registered, but it was expected to cover the area of the collimator and produce four distinguishable spots in the image. The positions of the collimator apertures are indicated by dashed circles. By changing the beam optics, it was possible to defocus and scan the beam through a variety of positions at the collimator surface which resulted in the various beam images shown in the Figs. 13(a)-13(d). However, under no circumstances was the lower hole visible in the images. The projection of a 3-mm diameter aperture on the righthand side is clearly distinguishable from the other objects. The same applies to the upper 2-mm diameter hole. Some ghost structures are visible around the middle 2-mm diameter hole. These can be caused by reflected particles at oblique incidence within the apertures but also by scattered secondary electrons due to aluminum surface aberrations. The distortion is subject to further analysis which can be based on future measurements with a thin foil replaced with a flat plate.
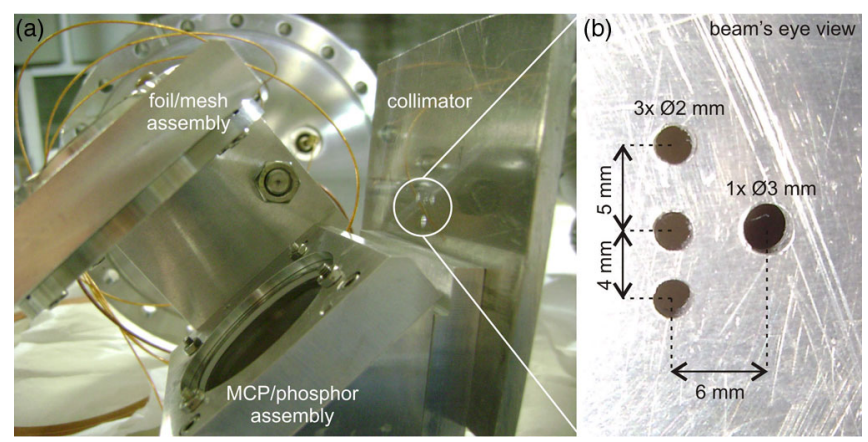

FIG. 12. Secondary emission monitor (a) and the collimator (b) as installed at INFN-LNS.

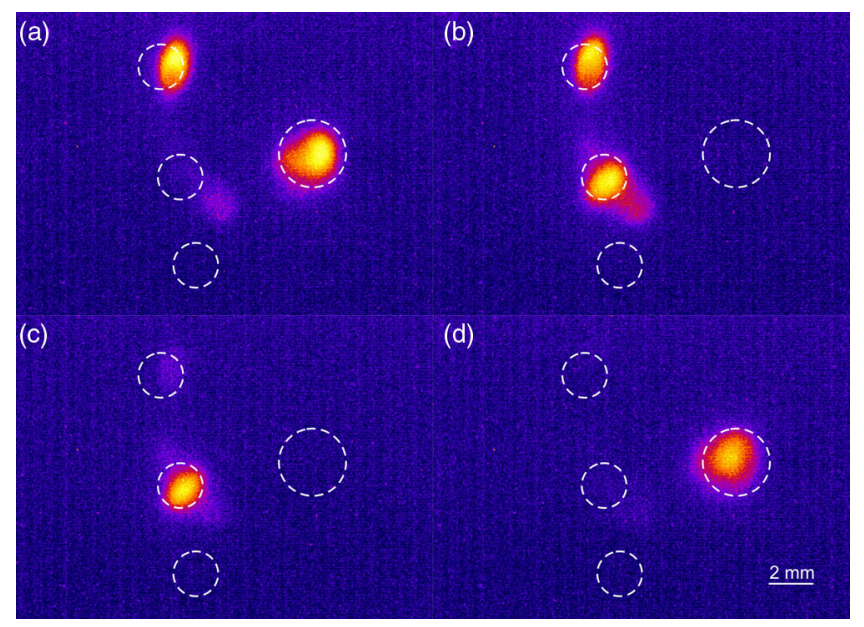

FIG. 13. Beam images obtained with the collimator for various beam steering settings.

Although the image does not perfectly resemble the shape of the collimator holes, an important conclusion can be made. The beam spots obtained during the experiment are of the same size as the apertures in the collimator, without additional blurring of $2-3 \mathrm{~mm}$ expected from theoretical calculations. This means that spatial resolution better than $2 \mathrm{~mm}$ can be obtained and the secondary emission model used in the simulations overestimates the energy and angular spread of guided electrons.

Further studies of a modernized secondary emission monitor equipped with a plate instead of the foil and a different type of a collimator are currently anticipated. The SEM is planned to be tested with antiprotons at the AEgIS experiment at CERN [30]. It is also intended to explore the applicability of the monitor to profile and emittance measurements with exotic ion beams at INFN-LNS.

\section{FARADAY CUP}

\section{A. Motivation}

Most beam intensity monitors used in particle accelerators have not been designed to measure femtoampere currents and need to be either pushed to their detection limits or replaced by other, more suitable devices known from particle physics experiments. Particle counters, such as scintillators or electron multipliers, can offer sufficiently high sensitivity, but get saturated or damaged if too high currents are delivered (e.g. [31,32]). They also require absolute calibration and quality check to ensure a constant response with time. Similarly, polycrystalline chemicalvapor-deposition diamond detectors show promising perspectives for ultralow current monitoring, but need to be calibrated and exhibit unstable leakage currents which vary with time, bias voltage, polarity, and dose [33]. It is therefore desirable to provide a simple and reliable solution which can be used to monitor low DC beam currents and sustain, or even measure, relatively high peak intensities. 
Faraday cups seem to fulfill all the requirements, but their ultimate detection limits have not been investigated in detail. These beam monitors are the easiest to operate and no simpler yet reliable technique can be applied for absolute current measurements. At low energies, the design of a Faraday cup does not have to be complex and high peak currents usually are not a problem, depending on cooling. With a careful design, picoampere currents can be measured, but it is a challenge to obtain satisfactory results in the femtoampere region [13]. The aim of this work was to investigate the detection limits of a customized Faraday cup and to optimize its design for low-energy, lowintensity proton and ion beams.

\section{B. Design considerations}

The most straightforward way to measure the intensity of a charged particle beam is to transform it to electric current in a conducting wire connected to an ammeter outside the vacuum tube. In this sense, a Faraday cup is an interface designed to collect all the primary and secondary charges and pass the resulting electrical signal to a transimpedance amplifier. A reliable Faraday cup is expected to collect all the charge carried by the primary beam, introduce very low noise, and work in a repetitive manner. However, resolution of a Faraday cup system is determined by the noise of the amplifier in dependence of its analog bandwidth. By limiting the bandpass of the system, the measurement's uncertainty can be reduced but at the cost of its applicability to time-varying beam pulses.

Primary charged particles can leave the detector either due to transmission through an insufficient thickness of the beam stopper or due to reflection from the Faraday cup surface. The first effect can be easily avoided because the average projected range of the USR beams in copper is about $1.5 \mu \mathrm{m}$ at $300 \mathrm{keV}$ and approximately $120 \mathrm{~nm}$ at $20 \mathrm{keV}$ [7]. This means that $\mathrm{keV}$ protons can be stopped even in a sufficiently thick foil. A solution to avoid backscattered projectiles leaving the monitor is less obvious. Beam energies at the USR fall into a range for which the validity of various analytical descriptions of scattering is limited [34]. The most satisfying solution can be provided by Monte Carlo techniques.

The TRIM Monte Carlo code [35] was used to calculate reflection of $\mathrm{keV}$-range protons from a beam stopper. Pencil beams at energies between 300 and $20 \mathrm{keV}$ impinging on a copper surface were simulated at normal incidence and at $75^{\circ}$ as measured from the normal to the target surface and shown in Fig. 14.

The total number of backscattered particles increased with increasing angle of incidence and decreased with increasing energy. The number of simulated primary protons varied between $10^{5}$ and $10^{7}$ in order to obtain statistically accurate results. At $300 \mathrm{keV}$, the fraction of backscattered protons with respect to the number of incident protons was $7.2 \times 10^{-4} \pm 12 \%$ at $0^{\circ}$ and

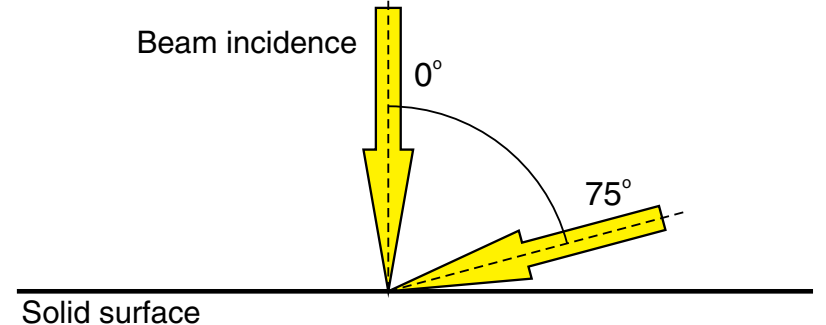

FIG. 14. Two angles of incidence for beams modeled in TRIM.

$4.7 \times 10^{-2} \pm 1.5 \%$ at $75^{\circ}$, whereas the values were significantly higher at $20 \mathrm{keV}$ and reached $2.4 \times 10^{-2} \pm$ $2.0 \%$ and $3.6 \times 10^{-1} \pm 0.5 \%$ at $0^{\circ}$ and $75^{\circ}$, respectively. Reflected protons carrying the highest energy were observed at a specular angle as presented in Fig. 15. This can be explained by single scattering from the surface which resulted in a small energy loss. At the same time, the most probable backscattered energy at oblique incidence was reduced to below $10 \mathrm{keV}$. The highest number of counts was observed around specular reflection at $20 \mathrm{keV}$, whereas this was not the case for $300 \mathrm{keV}$ protons. When incident at $75^{\circ}$, they penetrated a shallower layer of copper compared to $20 \mathrm{keV}$, but traveled at larger distances before
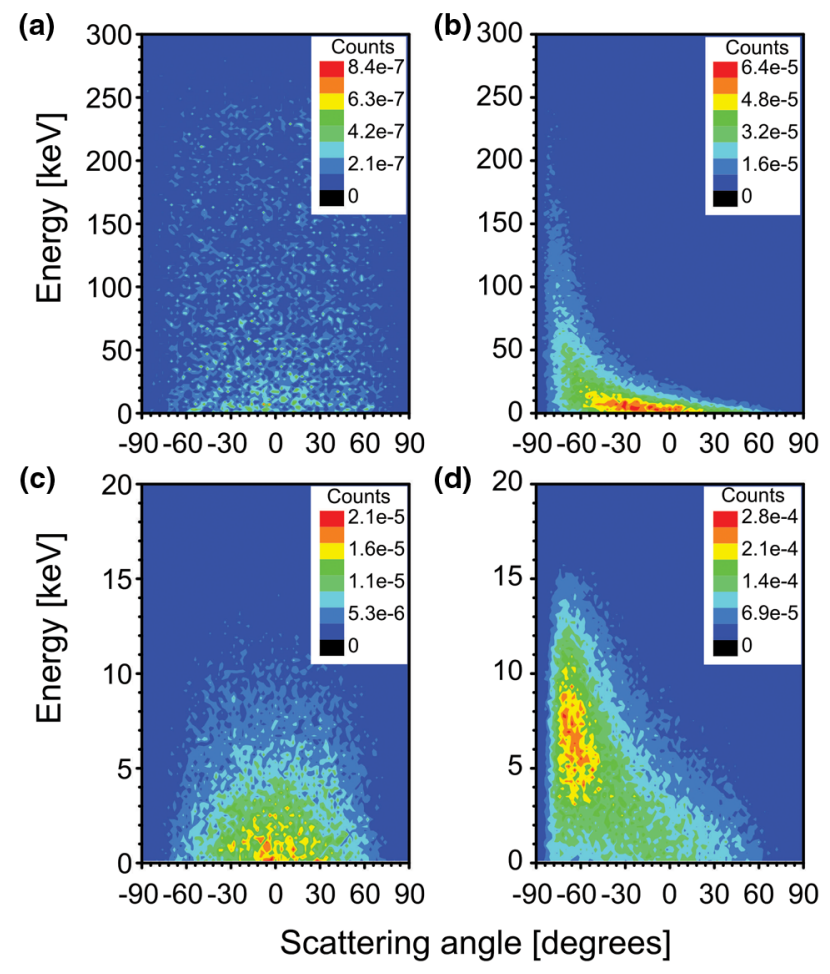

FIG. 15. Backscattered energy distribution as a function of scattering angle for $300 \mathrm{keV}$ protons impinging on a copper surface at $0^{\circ}$ and $75^{\circ}$ [(a) and (b), respectively] and $20 \mathrm{keV}$ protons impinging at $0^{\circ}$ and $75^{\circ}$ [(c) and (d), respectively]. The counts are given as a fraction of the number of simulated incident protons: (a) $1.8 \times 10^{7}$, (b) $1.4 \times 10^{6}$, (c) $1.5 \times 10^{6}$, and (d) $2.8 \times 10^{5}$. 
leaving the material. The number of protons that exited the target slowly increased to reach the maximum at the distance corresponding to their range in copper and then dropped down rapidly. This effect was due to multiple small-angle scattering and resulted in a different distribution of backscattered energy as a function of scattering angle. Because $20 \mathrm{keV}$ protons scatter much easier, they did not penetrate much of the copper target before leaving it, thus did not deposit energy in multiple small steps.

Several solutions can be applied in order to reduce the number of reflected protons. A simple plate is not sufficient to act as an effective beam collector and either additional side walls or a hollow block with a narrow entrance channel should be used. Backscatter depends on the atomic number of the target and a low atomic number material is preferred. Furthermore, a biased grid can be introduced to reduce the number of reflected protons, but it would affect the number of incoming projectiles as well.

In addition to backscattered particles, also secondary electrons carry a charge which should not leave the Faraday cup. Their characteristics have been extensively studied by many groups [22,36]. Secondaries ejected backwards can be stopped by means of an electric field applied at the entrance of the Faraday cup. Also a narrow channel can minimize the probability of particles escaping from the monitor.

In the case of antiproton beams, the use of the Faraday cup will be strongly limited. The reason for this is the creation of high-energy charged annihilation products which cannot be stopped in a compact detector. Despite this limitation, the Faraday cup will be an important diagnostics tool during the USR commissioning with protons. In addition, it can be used to compare the response to both, the proton and antiproton beams of similar intensities as well as to serve as a reference for relative current measurements with capacitive pickups.

A particle beam stopped in a Faraday cup can cause a temperature rise. The beam stopper is electrically insulated from its environment, thus heat transfer by conduction is strongly limited. Also convection in a vacuum system is negligible and the only considerable process of cooling down is thermal radiation. Considering an energy of $300 \mathrm{keV}$ and an instantaneous beam current of $1 \mu \mathrm{A}$, the peak beam power in the USR will be less than $300 \mathrm{~mW}$. It was demonstrated that no additional cooling system needs to be used if the beam power does not exceed some watts [37].

\section{Prototype design}

The design of the Faraday cup prototype is shown in Fig. 16. It consists of a cylindrical beam collector with a conical cutout and a suppressing ring electrode. Both are electrically isolated from each other and shielded against external influences by means of a grounded case. The suppressor and the shield are made of nonmagnetic

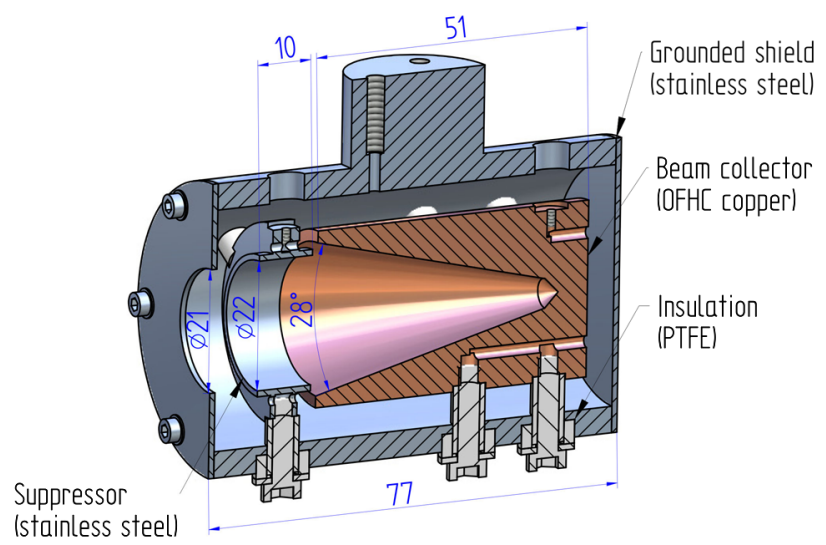

FIG. 16. Cross-section view of the Faraday cup design.

stainless steel, whereas the beam collector is made of oxygen-free high thermal conductivity (OFHC) copper. The entrance to the cup is $21 \mathrm{~mm}$ in diameter which defines the maximum beam size that can be detected by the device.

Protons backscattered from the copper beam stopper were modeled with a Monte Carlo code. The TRIM package could not be used in this case because it is only suitable for simulations of particles impinging on a flat surface. Instead, the beam stopper was modeled with GEANT4 [38]. A beam containing $5 \times 10^{5}$ protons was simulated to hit the concave surface of the copper block and the QGSP BERT HP physics list was used [38]. At $300 \mathrm{keV}$, only 1 proton escaped the cup, a negligible amount even with statistical fluctuations taken into account. At $20 \mathrm{keV}$, less than $0.2 \%$ particles were scattered back to the entrance and left the detector. Therefore, the current measurement by means of the proposed Faraday cup at the lowest energy of $20 \mathrm{keV}$ is underestimated by approximately $0.2 \%$. At $300 \mathrm{keV}$, backscattering is negligible and does not affect the observed beam intensity.

Secondary electron emission and suppression were modeled in SIMION and CST PARTICLE STUDIO [39]. The energy and angular distributions were defined according to [22]. With the suppressing electrode voltage above a few hundred volts, no secondary electrons were observed to leave the beam stopper.

For ultralow current measurements, low-noise triaxial cables are used. Double screening reduces the noise caused by ground loops and other parasitic effects. Such cables and feedthroughs, however, are not available for ultrahigh vacuum of about $10^{-11}$ mbar under which the Faraday cup will be operated. For this reason, a custom-made Kaptoninsulated coaxial cable with an additional stainless steel screen was prepared. The shielding is ensured by the stainless steel braid grounded at its both ends to the vacuum chamber. The guarding is ensured by the inner shield grounded on the side of the amplifier and left floating on the Faraday cup side. This way, the voltage potential of the signal conductor is very close to the voltage potential of the inner shield which is set to zero. 
To overcome the difficulties with measurements under various beam delivery schemes at the USR, a variable gain transimpedance amplifier DLPCA-200 from FEMTO [40] is used. It offers several gain and bandwidth settings. A gain of either $10^{6}$ or $10^{7} \mathrm{~V} / \mathrm{A}$ is sufficiently high for sub- $\mu \mathrm{A}$ bunched beam peak currents, whereas the rise/ fall time enables sampling the inner bunch structure. For sub-pA quasi-DC beams, the highest sensitivity can be achieved with $10^{11} \mathrm{~V} / \mathrm{A}$ and an internal low-pass filter with the upper cutoff frequency set to $10 \mathrm{~Hz}$. For even higher sensitivity, an LCA-2-10T FEMTO amplifier is used which provides a gain of up to $10^{13} \mathrm{~V} / \mathrm{A}$ and a narrow bandwidth as small as $0.1 \mathrm{~Hz}(-3 \mathrm{~dB})$. The drawback, however, is a longer rise/fall time which for $0.1 \mathrm{~Hz}$ is $5 \mathrm{~s}$. The maximum input current of $\pm 1 \mathrm{pA}$ at $10^{13} \mathrm{~V} / \mathrm{A}$ limits the dynamic range of the detection system.

\section{Experimental results}

The response of the Faraday cup, together with the secondary emission monitor described in Sec. III, was investigated with $\mathrm{keV}$ protons available at INFN-LNS. The experimental setup was already introduced in Fig. 7. Because of time constraints, only the LCA-2-10T amplifier with the lowest bandwidth of $0.1 \mathrm{~Hz}$ at $-3 \mathrm{~dB}$ was tested. The amplified signals were transferred to the control room and registered by a $1 \mathrm{GHz} 8$-bit oscilloscope, WS104MXsA WaveSurfer from LeCroy [41].

The influence of secondary electron emission on proton beam-current measurements was studied with various voltages applied to the suppressing electrode. The signal measured without suppression was $75 \%$ higher than for the fully suppressed secondaries. The ejection of electrons from the detector results in a higher positive charge in the beam collector and gives rise to the current flow in the system. The reading is therefore falsified by the amount of charge carried away by secondary electrons. As shown in Fig. 17, the effect is minimized above a certain suppression voltage and the actual beam current can be measured. Further tests were therefore performed at a fixed voltage of $-300 \mathrm{~V}$.

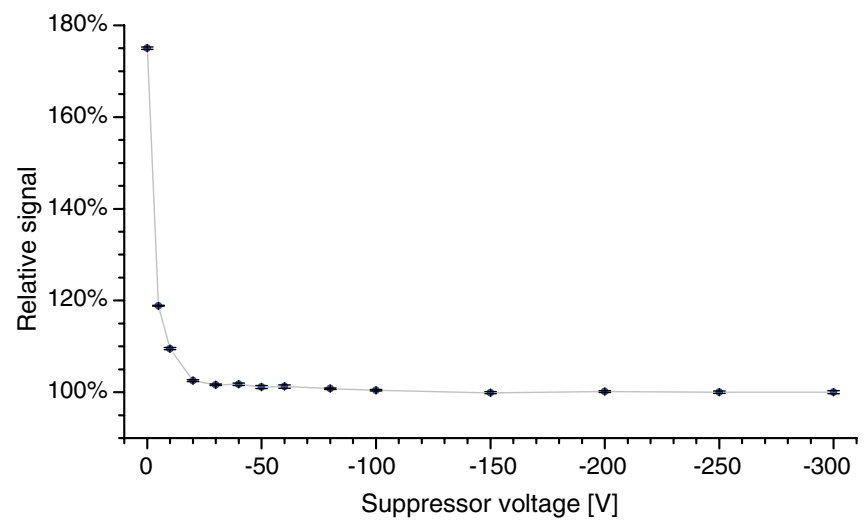

FIG. 17. Suppression of secondary electrons as a function of the voltage applied to the suppressing electrode.

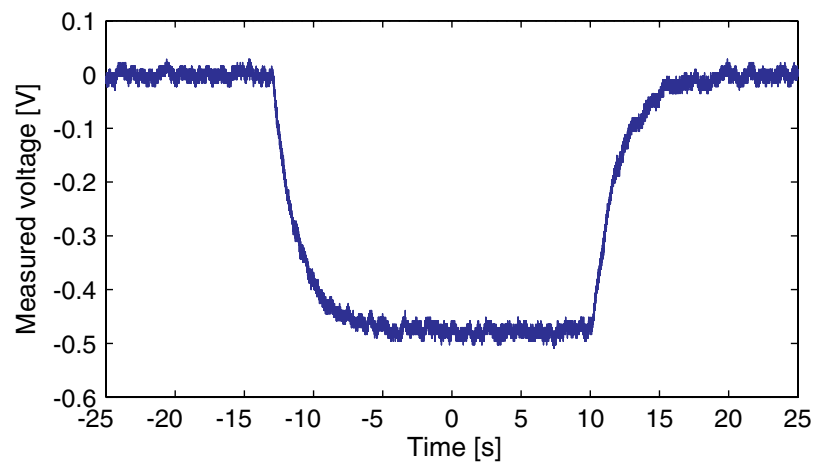

FIG. 18. Response of the Faraday cup to a chopped beam corresponding to about $500 \mathrm{fA}$ registered at $10^{12} \mathrm{~V} / \mathrm{A}$.

The response of the Faraday cup to DC beams and pulses of finite length was investigated. The beam delivered by the INFN-LNS injector was continuous and no rf buncher was available to modulate it. However, the primary Faraday cup installed on a pneumatic actuator was used as a beam chopper. It was moved in and out, and the resulting signals at various beam currents were registered to evaluate how the bandwidth and noise affect the performance of the Faraday cup. Figures 18 and 19 show observed chopped beams at two different current levels.

Figure 18 shows a waveform registered at $10^{12} \mathrm{~V} / \mathrm{A}$ gain and corresponds to a beam current of the order of $500 \mathrm{fA}$. The time needed to move the INFN-LNS beam stopper in or out was much shorter than $1 \mathrm{~s}$ and was small compared to the slow response of the LCA-2-10T amplifier. The $10 \%-90 \%$ rise time of the signals corresponds to $5 \mathrm{~s}$ as specified by the manufacturer for $0.1 \mathrm{~Hz}$ bandwidth. The peak-to-peak noise of about $40 \mathrm{fA}$ is present in the signal, but the rise and fall time of the pulse can be clearly noted.

The other example, given in Fig. 19, shows a waveform corresponding to a beam current of the order of $80 \mathrm{fA}$. The noise becomes a significant component and the fluctuations make it more difficult to pinpoint the beginning and the end of the beam pulse. The waveform was registered at

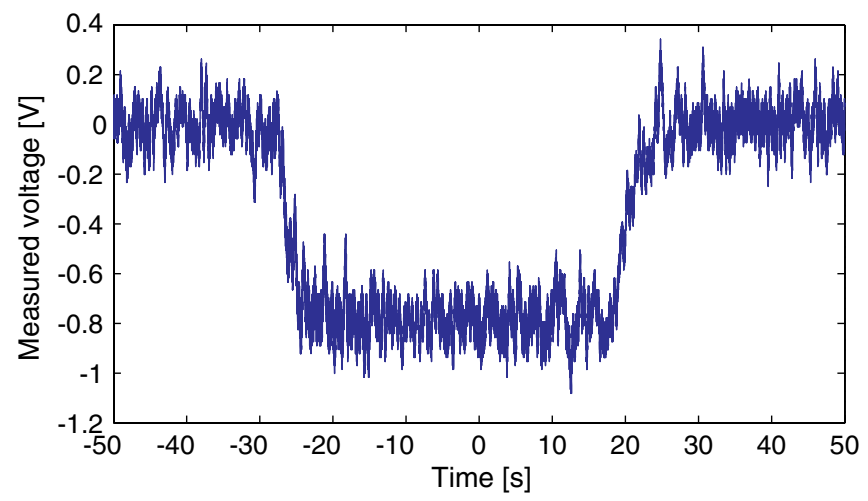

FIG. 19. Response of the Faraday cup to a chopped beam corresponding to about $80 \mathrm{fA}$ registered at $10^{13} \mathrm{~V} / \mathrm{A}$. 
$10^{13} \mathrm{~V} / \mathrm{A}$ gain, but no difference in the signal-to-noise ratio was observed as compared to $10^{12} \mathrm{~V} / \mathrm{A}$. This is because the amplification is done by the primary circuit related to $10^{12} \mathrm{~V} / \mathrm{A}$ and a second amplifier introduced at its output. Consequently, both signal and noise are amplified and the signal-to-noise ratio (SNR) is not improved in the second stage.

In order to identify possible noise sources affecting the performance of the monitor, the frequency spectra of the signals registered at various beam currents were calculated. Baseline measurements shown in Fig. 20 present the noise picked up by the system without the beam presence. Other beam-current levels are designated by only approximate values of 481 and $57 \mathrm{fA}$. The noise related to the main frequency of $50 \mathrm{~Hz}$ has been minimized, but other components are clearly visible in the spectra. The strongest influence on the monitor performance has a peak at around $13.5 \mathrm{~Hz}$. Additionally, a frequency component around $165 \mathrm{~Hz}$ with two sidebands is also registered by the Faraday cup. There is also a strong increase of noise below $4 \mathrm{~Hz}$.

The frequency components present in the spectrum shown in Fig. 20 can be due to several factors. The noise could be caused by parts prior to the amplifier input, such as vibrating vacuum pumps, but more likely by variation of the transimpedance gain, by electromagnetic inference between the amplifier and the oscilloscope, or by the oscilloscope itself. The transimpedance amplifier had an upper cutoff frequency of $0.1 \mathrm{~Hz}$ at $-3 \mathrm{~dB}$, but frequency components above this cutoff frequency were damped according to the filter ordering. However, the filter characteristics of the commercial amplifier were not provided by the manufacturer. Additionally, it would have been more

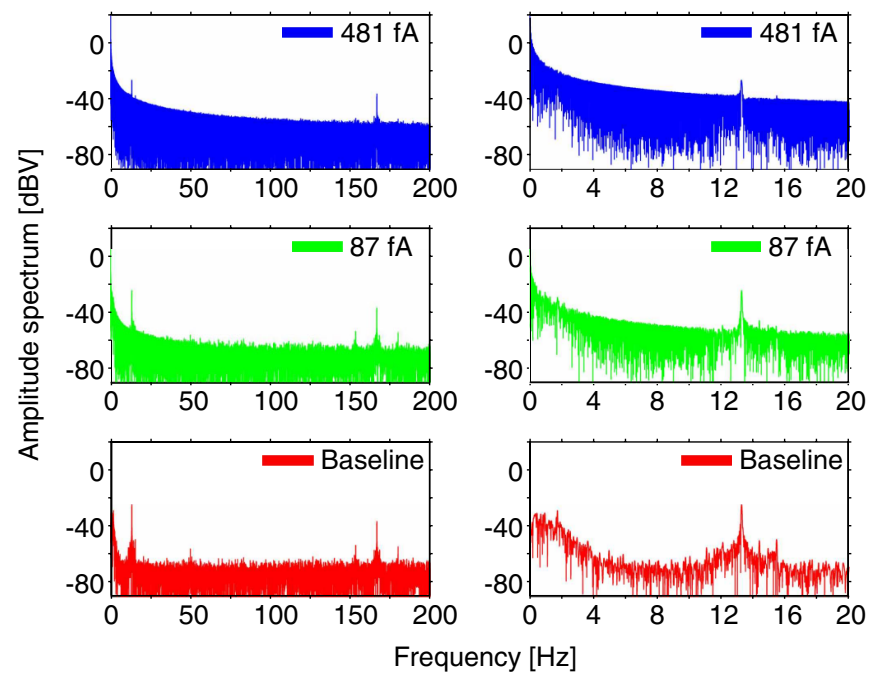

FIG. 20. Frequency spectra of the signals registered for various beam currents at INFN-LNS: $481 \mathrm{fA}$ (top), $87 \mathrm{fA}$ (middle), and no beam (bottom). The right column represents the same measurements as the left one but shows the low-frequency part in detail.

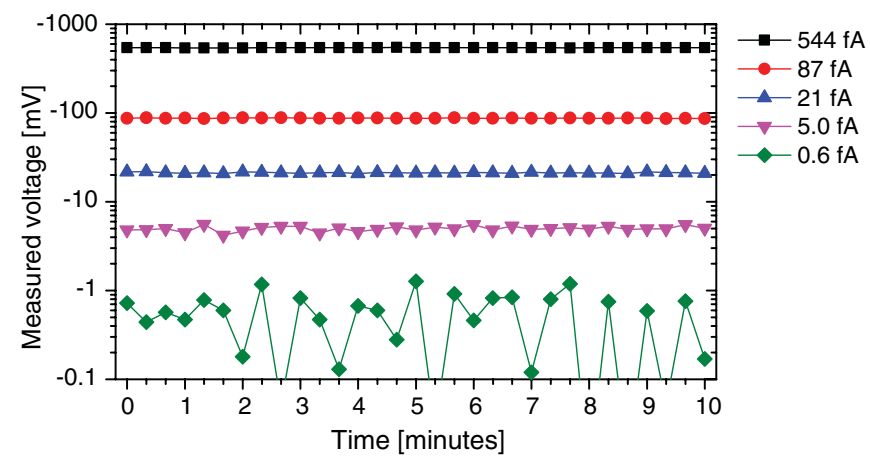

FIG. 21. Beam-current measurements at $10^{12} \mathrm{~V} / \mathrm{A}$ transimpedance gain for various proton beam intensities at INFN-LNS. Each point is averaged over $20 \mathrm{~s}$.

appropriate to use a spectrum analyzer suited for low frequencies as compared to a fast sampling oscilloscope with low voltage resolution. Further tests and setup optimization can still be performed to minimize the intrinsic noise of the system.

To investigate the detection limits of the Faraday cup prototype setup as prepared for the beam time at INFN-LNS, baseline shift measurements with additional averaging were performed. First, the background baseline without the beam was determined. Second, a DC component of the signal obtained with the beam was derived from the average over 20 seconds. The acquisition time was chosen to correspond to the slow extraction time of the order of tens of seconds. Various intensities were obtained by allowing only a fraction of protons to reach the monitor. Each time the current was set to an arbitrary level, the beam presence was verified by both, the USR Faraday cup and the secondary emission monitor. Figure 21 shows various beam intensities as measured by the current monitor, from the level of about $500 \mathrm{fA}$ to no beam at all. In order to study the reproducibility, the measurements were recorded over 10 minutes in each case and no drift of the results was observed. The currents seen in the figure correspond to $544 \mathrm{fA} \pm 11 \mathrm{fA}$ (black curve), $87 \pm 2 \mathrm{fA}$ (red curve), $21 \mathrm{fA} \pm 0.5 \mathrm{fA}$ (blue curve), $5.0 \mathrm{fA} \pm 0.3 \mathrm{fA}$ (pink curve), and the measurement without the beam with an average offset of $0.6 \mathrm{fA} \pm 0.3 \mathrm{fA}$ (green curve). The results demonstrate that DC beam currents as low as a few femtoamperes can by measured by the Faraday cup designed for the USR.

\section{CAPACITIVE PICKUP}

\section{A. Motivation}

A capacitive pickup is the device of choice for beam position measurements in most particle accelerators due to their nondestructive character. At relativistic velocities, the charge induced on a metal electrode is a direct image of the bunches and the description of the system response becomes very simple [42]. Also, intense beams lead to 
sufficiently strong signals that can be easily detected and deliver precise information on the beam displacement from the reference orbit as well as on the structure of individual bunches.

The USR puts challenging demands on the beam pickups which should be capable of measuring beam position at both, low intensities and low velocities. The number of antiprotons in the USR is expected to result in signals that are close to the noise level of state-of-the-art electronics. A low-noise signal processing system with a reduced bandwidth is therefore required. Also, the velocities of the decelerated particles in the USR complicate the already difficult task of beam position determination. The low energies correspond to only a fraction of the speed of light and the induced charge distribution is not a direct image of the beam any more. It becomes dependent on the bunch length, repetition frequency, and transverse displacement. All these difficulties need to be carefully addressed in order to provide a diagnostic system capable of monitoring the low-intensity, low-energy antiproton beams at the USR.

\section{B. Principle of operation}

A charged particle beam passing near metal electrodes generates image currents which can be coupled via amplifiers for further processing. For bunches considerably longer than the pickup electrodes and high-impedance amplifiers, the pickup signal is a direct image of the bunch [42] and can be described by

$$
U(t)=\frac{l}{C \cdot v} \cdot I(t),
$$

where $U(t)$ is the detected voltage, $l$ is the pickup length, $C$ is its capacity to ground, $v$ is the beam velocity, and $I(t)$ is the beam current.

In hadron storage rings, a diagonally cut capacitive pickup is a commonly used beam position monitor. Such a shape is of particular interest, because the signal amplitude becomes proportional to the fraction of the beam covered by the electrodes as projected on a given plane. In turn, the response of the pickup changes linearly with the beam position. The displacement $x$ of the center of mass of the beam with respect to the center of the vacuum tube can be expressed as the difference of the electrodes signals $\Delta U$ normalized to their sum $\Sigma U$ :

$$
x=k \cdot \frac{\Delta U}{\Sigma U}+\delta
$$

where $k$ is a linear scaling factor and $\delta$ is the pickup offset which represents the misalignment of the electrical center with respect to the geometrical center of the pickup. The scaling factor is proportional to the pickup radius $r$ and depends on the parasitic coupling capacitance $C_{C}$ between the electrodes:

$$
k=r \cdot\left(1+2 \cdot \frac{C_{C}}{C}\right)
$$

The smallest value of $k$ corresponds to the highest sensitivity and, as a result, a small beam displacement generates a large difference signal. The parasitic coupling between the adjacent electrodes increases $k$ and limits the sensitivity of the pickup. A very low coupling capacity is therefore desirable.

The above considerations are based on the assumption that the charge distribution induced on the pickup electrodes is a direct image of a moving bunch which is true only for ultrarelativistic particles [43,44]. At velocities close to the speed of light, the electric field lines are perpendicular to the bunch direction due to the Lorentz contraction of the longitudinal field components. Consequently, bunches induce a wall image current which has the same time structure as the beam. However, at low beam velocities, the longitudinal component of the electric field does not vanish and the image charge distribution is broader than the bunch length. Furthermore, its width depends also on the beam distance to the metallic wall and a nonlinear response of the pickup is expected. A way to analytically treat such signals is described in detail in [44].

\section{Prototype design}

The USR pickup consists of two pairs of long metal electrodes. Each pair measures the beam position only in one direction and two pairs, rotated by $90^{\circ}$ with respect to each other, are required. It is a cylindrical device with diagonally cut electrodes and additional separating rings to reduce the parasitic coupling capacitance. The monitor is shown in Fig. 22.

The electrodes and rings are made of nonmagnetic stainless steel, whereas the outer shield is made of aluminum to reduce the overall weight of the monitor. The diagonally cut electrodes are $100 \mathrm{~mm}$ in length and $100 \mathrm{~mm}$ in diameter. The grounded separating rings can be removed from the setup, if needed. Their length is approximately $13 \mathrm{~mm}$ and the inner diameter matches that of the

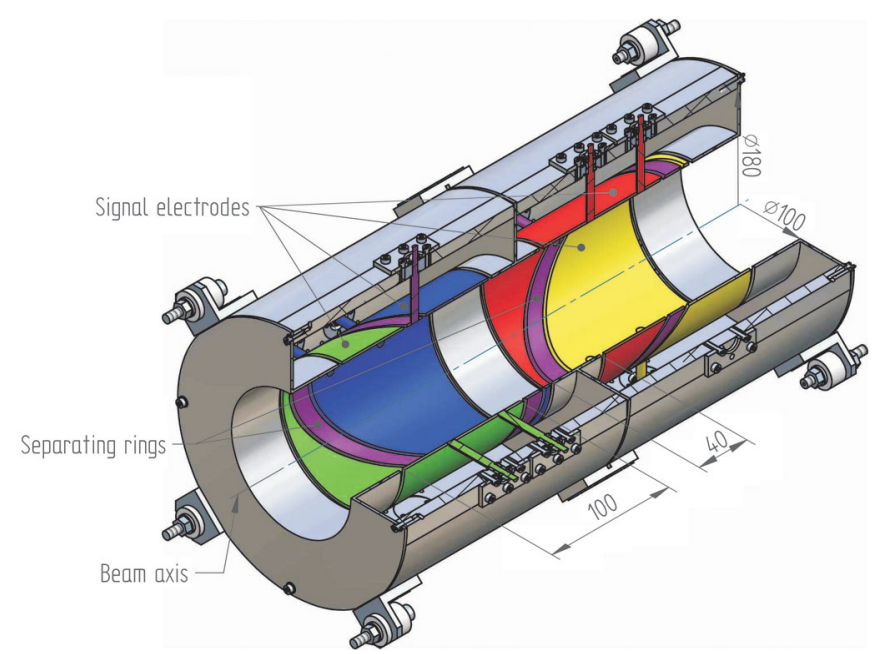

FIG. 22. Mechanical design of the capacitive pickup monitor. 
electrodes. The spacing between the rings and the electrodes is about $2 \mathrm{~mm}$.

The outer shield ensures shielding against electromagnetic noise. It is on a separate "clean" ground and is electrically isolated from the vacuum vessel in which the monitor is installed. In order to avoid distortion of the electric field in the vicinity of the monitor edges, the inner diameter of the pickup, $100 \mathrm{~mm}$, is the same as the diameter of the USR beam pipe. In addition, grounded rings are introduced at both ends and in the middle of the monitor. The outer ones minimize the effect of the transition between the pickup and the vacuum chamber walls, whereas the inner reduces the coupling between $x$ and $y$ planes. The length of the grounded rings was limited to $40 \mathrm{~mm}$ because of spatial constraints in the USR.

The pickup signal is coupled via a high-input impedance amplifier and passed to a low-pass filter before digitization. The analogue filtering is optional because the signal frequency range is much smaller than the sampling rate of the analog-to-digital converter. Nevertheless, such a filter can be useful in the case of a high frequency distortion of the signal resulting in artificial frequencies due to aliasing. The processing and beam position determination are then performed in a digital domain by a customized LABVIEW [45] application.

The signals generated on the electrodes are fed to lownoise, high-input impedance amplifiers, SA-220F5 from NF Corporation [46], and digitized by a commercial digitizer, GaGe Razor CompuScope CS1642 [47], equipped with 4 channels with $128 \mathrm{MB}$ on-board acquisition memory. A LABVIEW application was prepared to control the digitizer and to display, analyze, and save the signals from the pickup electrodes.

\section{Experimental results}

The most common approach to test and calibrate a beam position monitor is the use of a current-carrying wire stretched inside the device under test [48]. Such a wire simulates the presence of a real particle beam. The technique can be used in a simple bench setup independently from a high vacuum environment and limited beam time at an accelerator. The stretched-wire inside the monitor corresponds to a coaxial cable arrangement in which only the transverse electromagnetic waves propagate, i.e., there is no component of the electric and magnetic fields in the direction of propagation of the wave [49]. This limits the use of the method to ultrarelativistic beams, because the longitudinal component of the electric field does not vanish at low beam velocities. Relevant correction can be found as shown in the further parts of this paper. A test stand was built to calibrate the USR pickup and is shown in Fig. 23.

The response of the monitor to a current-carrying wire was investigated and the presence of the separating rings had a significant impact on the results. Better position sensitivity was observed for the configuration with the

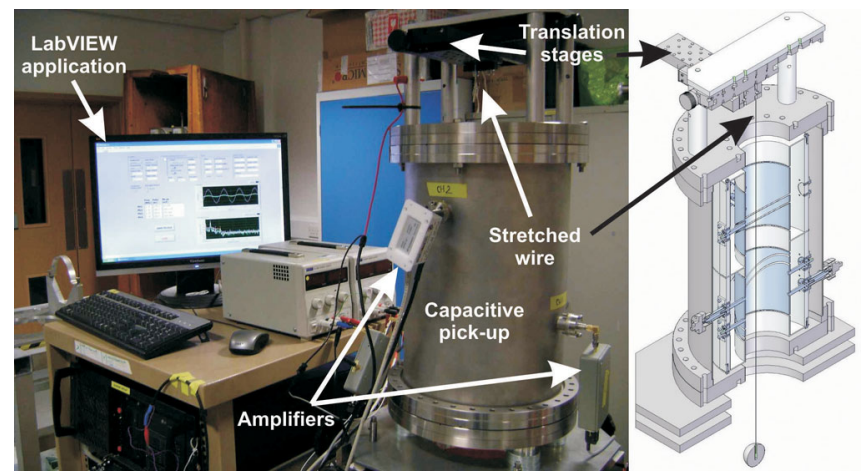

FIG. 23. Capacitive pickup test stand with a current-carrying wire.

rings and was almost 20\% higher than for the setup without the rings. On the other hand, the voltage measured without rings was higher by about $20 \%$ as compared to the pickup with the rings added. There is, therefore, a trade-off between the decoupling of the pickup electrodes and their detection sensitivity.

Table II summarizes the obtained scaling factors $k$ together with the determined offsets $\delta$ of the electrical center with respect to the geometrical center. The values of $k$ for a given monitor configuration were the same for $x$ and $y$ axes within less than $1 \%$. No change in the results was observed as a function of frequency in the investigated range of $400 \mathrm{kHz}-2 \mathrm{MHz}$. The uncertainties in $k$ correspond to position uncertainties of the order of $0.1 \mathrm{~mm}$. The electrical offsets were zero within the uncertainty of $0.1 \mathrm{~mm}$ for the setup with separating rings, whereas they became nonnegligible for the pickup without the rings. This may have been caused by a higher coupling between the opposite electrodes when no separating ring is present. The diagonal cut configuration is not symmetric with respect to the beam arrival time and this may be reflected in the measurements. However, the determined beam position can be corrected for the reported values.

The beam velocities at the USR are much smaller than the speed of light, thus deviations from the ultrarelativistic case need to be estimated. An analytical solution to this problem is discussed in detail in [44] and was applied to the pickup with $50 \mathrm{~mm}$ radius and bunching frequencies $f_{\text {rf }}$ corresponding to those possible at the USR. It was assumed that the beam position is determined by the pickup

TABLE II. Scaling factors $k$ and offsets $\delta$ of the electrical center in $x$ and $y$ for the pickup with and without the separating rings.

\begin{tabular}{lcc}
\hline \hline & Without separating rings & With separating rings \\
\hline$k_{x}[\mathrm{~mm}]$ & $71.5 \pm 0.1$ & $59.8 \pm 0.1$ \\
$k_{y}[\mathrm{~mm}]$ & $70.8 \pm 0.5$ & $60.3 \pm 0.3$ \\
$\delta_{x}[\mathrm{~mm}]$ & $-0.3 \pm 0.2$ & $-0.1 \pm 0.1$ \\
$\delta_{y}[\mathrm{~mm}]$ & $-0.4 \pm 0.2$ & $0.1 \pm 0.1$ \\
\hline \hline
\end{tabular}


response to a single frequency, i.e., narrowband signal processing locked-in at $f_{\text {rf }}$ is used. Also a $\cos ^{2}$-like current modulation is considered, thus the bunch length is simply the inverse of the bunching frequency. Figure 24 shows the discrepancy between the beam position determined at various $f_{\text {rf }}$ values and the actual beam position. Only integer multiples $h$ of the revolution frequency $f_{\text {rev }}$ are allowed in a storage ring, i.e. $f_{\mathrm{rf}}=h \cdot f_{\mathrm{rev}}$. At $20 \mathrm{keV}$, the nonlinear effect becomes stronger at lower frequencies, but is similar for both USR energies if the same harmonic numbers $h$ are compared. The chosen harmonic number $h=10$ leads to a beam position determination error smaller than $0.02 \mathrm{~mm}$, thus the correction can be omitted. Should the short bunches be observed in the wide bandwidth, the low- $\beta$ effect will have to be taken into account.

The performance of the pickup was analyzed also in terms of signal and noise. The anticipated number of $2 \times 10^{7}$ particles circulating in the USR at $20 \mathrm{keV}$ corresponds to the average beam current of approximately $100 \mathrm{nA}$. For the pickup electrode with a length of $100 \mathrm{~mm}$ and capacitance of $80 \mathrm{pF}$, the expected sum and difference signals are then $\Sigma U=95 \mu \mathrm{V}$ and $\Delta U=$ $1.6 \mu \mathrm{V}$ for $1 \mathrm{~mm}$ beam displacement. The values are calculated according to Eqs. (1) and (2), but can be higher depending on the applied beam cooling and bunch compression [50]. A system capable of monitoring individual bunch structures at the USR would require a bandwidth of at least $20 \mathrm{MHz}$. With the unavoidable noise of about $1 \mathrm{nV} / \sqrt{\mathrm{Hz}}$, this corresponds to a signal-to-noise ratio (SNR) much less than 1. In order to observe bunch by bunch variations, the bandwidth could be reduced to about $2 \mathrm{MHz}$ which results in SNR of about 1 . Turn by turn diagnostics requires approximately $200 \mathrm{kHz}$ bandwidth which is more likely to be possible at the USR. However, monitoring of an average beam trajectory is sufficient for the operation of the machine and closed-orbit measurements can be performed. This makes it possible to apply

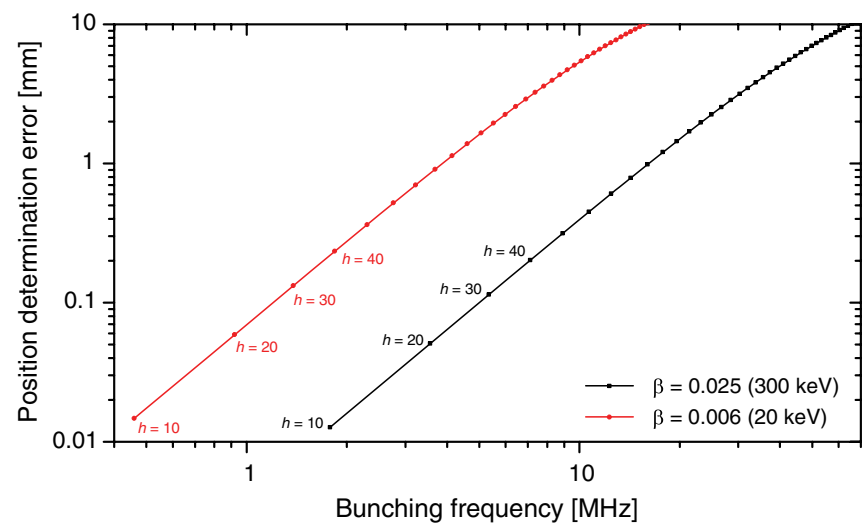

FIG. 24. Beam position determination error as a function of bunching frequency at $\beta=0.025$ (300 keV) and $\beta=0.006$ $(20 \mathrm{keV}$ ) for a pickup with $50 \mathrm{~mm}$ radius and a single frequency response at $f_{\text {rf }}=h \cdot f_{\text {rev }}$. a narrowband signal processing, necessary to minimize the noise present in the system and improve resolution below $1 \mathrm{~mm}$.

The uncertainties due to overall noise, mechanical accuracy, and signal digitization were analyzed in detail. The largest position determination error can be caused by a tilt of the monitor with respect to the beam axis, but can be corrected for, if known. The performance of the pickup is limited also by the resolution of state-of-the-art digitizers if the sum and difference signals are calculated after digitization. With the effective number of bits $b=14$, the position determination uncertainty cannot be smaller than $\pm 0.1 \mathrm{~mm}$. This is sufficient for the USR where also very spread out beams are to be localized within the $100-\mathrm{mm}$ aperture beam tube. It means that the accuracy of $\pm 0.1 \%$ can be achieved.

\section{CONCLUSIONS AND PERSPECTIVES}

A set of diagnostic instrumentation for low-energy, low-intensity charged particle beams was developed and tested with beams of $\mathrm{keV}$ energies and femtoampere range currents. The detectors include beam-profile monitors based on scintillating screens and secondary electron emission, a sensitive Faraday cup, and a capacitive pickup. Although the devices were primarily developed for the USR at FAIR, they can find application at other low-energy, low-intensity accelerators, storage rings, and beam lines.

For beam-profile measurements in the injection and extraction lines, a foil-based secondary emission monitor was designed. It was demonstrated that beam currents of a few femotamperes can be observed, yet no detection limits were reached. It was also shown that there are no advantages in using a thin foil for proton or ion imaging and a plate can be employed instead. This should rule out any problems related to electrostatic forces acting on a thin film. Additionally, a collimator was used and a spatial resolution of at least $2 \mathrm{~mm}$ was demonstrated. However, the recorded images indicate that further tests may reveal better performance. The images of $2 \mathrm{~mm}$ holes yielded higher spatial resolution than predicted in theoretical estimates.

Alternatively, CsI:Tl screens offer sufficiently high sensitivity to low-energy, low-intensity beams. It was demonstrated that it is possible to measure currents even in the fA range corresponding to about $10^{4}$ particles per second at $200 \mathrm{keV}$. For $50 \mathrm{keV}$ beams, the sensitivity drops down and is about 4 times lower. Additionally, an absolute light yield calibration technique can be applied to estimate the beam current of the impinging proton beams.

For measuring intensities of injected and extracted beams, a sensitive Faraday cup will be used. The prototype equipped with a commercial amplifier with a gain of $10^{12} \mathrm{~V} / \mathrm{A}$ and a bandwidth of $0.1 \mathrm{~Hz}$ yielded the peakto-peak noise of about $40 \mathrm{fA}$ and further averaging over 20 seconds was applied. It was demonstrated that beam 
currents as low as $5.0 \mathrm{fA} \pm 0.3 \mathrm{fA}$ can be measured in a reproducible manner.

For nondestructive beam position/closed-orbit measurements at the USR, at least four capacitive pickups will be installed in the ring. The diagonally cut electrodes guarantee linear response of the system to beam displacements within the required range of $\pm 40 \mathrm{~mm}$. It was demonstrated that the nonlinear effects caused by low beam velocities are not a problem in the USR as long as bunching frequencies $f_{\text {rf }}$ are low and a narrowband signal processing is used. The chosen harmonic number $h=10$ results in a beam position determination error smaller than $0.02 \mathrm{~mm}$, thus the low- $\beta$ correction can be omitted. Should the short bunches be observed in the wide bandwidth, the low- $\beta$ effect will have to be taken into account.

The Faraday cup will provide information on the proton or ion beam intensity, but can also be used to calibrate the response of the pickups. This way, their applicability can be extended to nondestructive proton and antiproton beamcurrent monitoring. For nondestructive acquisition of transverse profiles with sub-mm resolution instead, the use of a supersonic gas jet target based monitor is envisaged [11].

\section{ACKNOWLEDGMENTS}

The authors would like to thank Juan Luis FernandoHernandez of the Cockcroft Institute/ASTeC/STFC for performing FLUKA simulations for the secondary emission monitor studies, Germano Bonomi of the University of Brescia/AEgIS collaboration for performing GEANT4 simulations for the Faraday cup studies, Andras Paal of the Manne Siegbahn Laboratory for designing and manufacturing the customized low-noise amplifiers for the capacitive pickup, the CI/ASTeC design office, and the INFN-LNS accelerator and ion source staff, as well as other involved QUASAR Group members for their strong support. The generous support of the European Commission under Contract No. PITN-GA-2008-215080, Helmholtz Association of National Research Centers (HGF) and GSI Helmholz Center for Heavy Ion Research (GSI) under Contract No. VH-NG-328, and STFC Cockcroft Institute Core Grant No. ST/G008248/1 are acknowledged.

[1] FLAIR: Facility for Low-Energy Antiproton and Ion Research, http://www.flairatfair.eu.

[2] C. P. Welsch, M. Grieser, J. Ullrich, and A. Wolf, Nucl. Instrum. Methods Phys. Res., Sect. A 546, 405 (2005).

[3] LSR Low-energy Storage Ring Technical Design Report, Version 1.3, Manne Sieghban Laboratory, Physics Department, Stockholm University, 2011.

[4] 1st DITANET Topical Workshop: Low Current, Low Energy Beam Diagnostics, Hirschberg-Großsachsen, 2009 [http://www-bd.gsi.de/ditanet/].
[5] T. Sieber, H. Fadil, M. Grieser, R. von Hahn, and A. Wolf, in Proceedings of the 10th European Particle Accelerator Conference, Edinburgh, Scotland, 2006 (Edinburgh, Scotland, 2006).

[6] V. Chohan, M. Angoletta, M. Ludwig, O. Marqversen, P. Odier, F. Pedersen, G. Tranquille, U. Raich, L. Soby, and T. Spickermann, in Proceedings of the Particle Accelerator Conference, Chicago, IL, 2001 (Chicago, 2001).

[7] M. J., Berger, J. S. Coursey, M. A. Zucker, and J. Chang, ESTAR, PSTAR, and ASTAR: computer programs for calculating Stopping-Power and range tables for electrons, protons, and helium ions [http://physics.nist.gov/Star].

[8] P. Forck, Lecture Notes on Beam Instrumentation and Diagnostics (Joint University Accelerator School, Archamps, 2011).

[9] Low Level Measurements Handbook, Precision DC Current, Voltage, and Resistance Measurements (Keithley Instruments Inc., 2004), 6th ed. [http://www.keithley.com/ knowledgecenter/knowledgecenter_pdf/LowLevMsHandbk .pdf]

[10] M. Inokuti, Int. J. Rad. Appl. Instrum. D 16, 115 (1989).

[11] M. Putignano, K.-U. Kühnel, C.-D. Schröter, and C. P. Welsch, Hyperfine Interact. 194, 189 (2009).

[12] L. Cosentino and P. Finocchiaro, Nucl. Instrum. Methods Phys. Res., Sect. B 211, 443 (2003).

[13] J. Harasimowicz, L. Cosentino, P. Finocchiaro, A. Pappalardo, and C.P. Welsch, Rev. Sci. Instrum. 81, 103302 (2010).

[14] G. Ciavola, M. Castro, L. Celona, F. Chines, S. Gammino, and S. Marletta, Nucl. Instrum. Methods Phys. Res., Sect. A 382, 192 (1996).

[15] G. Drobychev et al. (AEgIS Collaboration), Reports No. CERN-SPSC-2007-017 and No. CERN-SPSC-P334, 2007.

[16] M. Hori (private communication).

[17] G. B. Andresen et al. (ALPHA Collaboration), Rev. Sci. Instrum. 80, 123701 (2009).

[18] K. Kruglov, L. Weissman, P. Van den Bergh, A. Andreyev, M. Huyse, and P. Van Duppen, Nucl. Instrum. Methods Phys. Res., Sect. A 441, 595 (2000).

[19] L. Badano, O. Ferrando, T. Klatka, M. Koziel, G. Molinari, and M. Pezzetta, IEEE Trans. Nucl. Sci. 51, 2990 (2004).

[20] S. Møller, E. Uggerhøj, H. Bluhme, H. Knudsen, U. Mikkelsen, K. Paludan, and E. Morenzoni, Phys. Rev. A 56, 2930 (1997).

[21] SIMION: ion and electron optics simulator [http://simion .com].

[22] D. Hasselkamp, Particle Induced Electron Emission II (Springer-Verlag, Berlin, 1992).

[23] D. Hasselkamp and A. Scharmann, Surf. Sci. 119, L388 (1982).

[24] H. Imao, H. A. Torii, Y. Nagata, H. Toyoda, T. Shimoyama, Y. Enomoto, H. Higaki, Y. Kanai, A. Mohri, and Y. Yamazaki, AIP Conf. Proc. 1037, 311 (2008).

[25] G. Battistoni, S. Muraro, P. R. Sala, F. Cerutti, A. Ferrari, S. Roesler, A. Fasso, and J. Ranft, The FLUKA code: Description and Benchmarking, AIP Conf. Proc. No. 896 (AIP, New York, 2007), pp. 31-49. 
[26] A. Ferrari, P. R. Sala, A. Fasso, and J. Ranft, Reports No. CERN-2005-10, No. INFN/TC_05/11, and No. SLAC-R-773, 2005.

[27] Beam Imaging Solutions INC. [http://www.beamimaging .com].

[28] W. S. Rasband, IMAGEJ, U.S. National Institutes of Health, Bethesda, Maryland, USA [http://rsb.info.nih.gov/ij/].

[29] V. Birman, Plate Structures (Springer, New York, 2011).

[30] M. Doser, J. Phys. Conf. Ser. 199, 012009 (2010).

[31] C. Bal, E. Bravin, T. Lefevre, R. Scrivens, and M. Taborelli, in Proceedings of DIPAC 2005 (Lyon, France, 2005).

[32] J. Krider, Fermilab PBAR Note \#444 (1986).

[33] E. Griesmayer, H. Pernegger, D. Dobos, F. Wenander, J. Bergoz, H. Bayle, H. Frais-Kölbl, J. Leinweber, and T. Aumeyr, Report No. CERN-BE-Note-2009-028, 2009.

[34] Vukanić, R. Janev, and D. Heifetz, Nucl. Instrum. Methods Phys. Res., Sect. B 18, 131 (1986).

[35] J. Ziegler, "Particle Interactions with Matter" [http:// www.srim.org].

[36] M. Rösler, W. Brauer, J. Devooght, J.-C. Dehaes, A. Dubus, M. Cailler, and J.-P. Ganachaud, Particle Induced Electron Emission I (Springer-Verlag, Berlin, 1991).

[37] P. Strehl, Beam Instrumentation and Diagnostics (Springer, Berlin, 2006).
[38] S. Agostinelli, Nucl. Instrum. Methods Phys. Res., Sect. A 506, 250 (2003).

[39] Computer Simulation Technology CST Studio 2010 [http://www.cst.com].

[40] FEMTO Messtechnik GmbH [http://www.femto.de].

[41] LeCroy Ltd. [http://www.lecroy.com].

[42] P. Forck, P. Kowina, and D. Liakin, in CERN Accelerator School: Beam Diagnostics, Dourdan, France, 2009.

[43] J. H. Cupérus, Nucl. Instrum. Methods 145, 219 (1977).

[44] R. E. Shafer, AIP Conf. Proc. 319, 303 (1994).

[45] National Instruments LABVIEW [http://www.ni.com/ labview/].

[46] NF Corporation [http://www.nfcorp.co.jp/english/index .html].

[47] Gage Applied Technologies, Inc. [http://www.gage-applied .com].

[48] A. Chao, Handbook of Accelerator Physics and Engineering (World Scientific, Singapore, 1999).

[49] V. V. Sarwate, Electromagnetic Fields and Waves (Bohem Press, Noida, 1993).

[50] M. Grieser, R. Bastert, K. Blaum, H. Buhr, R. von Hahn, M. B. Mendes, R. Repnow, and A. Wolf, Proceedings of the Conference on Heavy Ion Accelerator Technology, Venice, 2009. 